\title{
ARTICLE
}

Infectious medicine, virology

\section{Risk factors for death in 1859 subjects with COVID-19}

\author{
Lei Chen ${ }^{1} \cdot$ Jianming $\mathrm{Yu}^{1} \cdot$ Wenjuan $\mathrm{He}^{1} \cdot \mathrm{Li} \mathrm{Chen}^{2} \cdot \mathrm{Guolin}_{\mathrm{Yuan}}^{3} \cdot$ Fang Dong ${ }^{4}$ Wenlan Chen ${ }^{1} \cdot \mathrm{Yulin}^{\mathrm{Cao}}{ }^{1} \cdot$

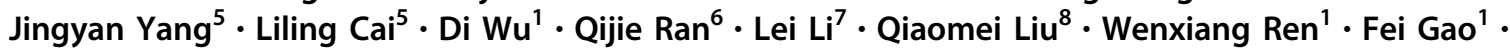 \\ Hongxiang Wang ${ }^{2} \cdot$ Zhichao Chen $^{1} \cdot$ Robert Peter Gale ${ }^{9} \cdot$ Qiubai Li $\mathbb{1}^{1} \cdot \mathrm{Yu} \mathrm{Hu} \mathbb{( i )}^{1}$
}

Received: 19 May 2020 / Revised: 2 June 2020 / Accepted: 3 June 2020 / Published online: 16 June 2020

(c) The Author(s), under exclusive licence to Springer Nature Limited 2020

\begin{abstract}
We studied 1859 subjects with confirmed COVID-19 from seven centers in Wuhan 1651 of whom recovered and 208 died. We interrogated diverse covariates for correlations with risk of death from COVID-19. In multi-variable Cox regression analyses increased hazards of in-hospital death were associated with several admission covariates: (1) older age (HR $=1.04$; 95\% Confidence Interval [CI], 1.03, 1.06 per year increase; $P<0.001)$; (2) smoking (HR = 1.84 [1.17, 2.92]; $P=0.009$ ); (3) admission temperature $\mathrm{per}^{\circ} \mathrm{C}$ increase (HR $=1.32$ [1.07, 1.64]; $P=0.009$ ); (4) $\log _{10}$ neutrophil-to-lymphocyte ratio (NLR; $\mathrm{HR}=3.30[2.10,5.19] ; P<0.001) ;(5)$ platelets per $10 \mathrm{E}+9 / \mathrm{L}$ decrease $(\mathrm{HR}=0.996[0.994,0.998] ; P=0.001) ;(6)$ activated partial thromboplastin (aPTT) per second increase (HR $=1.04$ [1.02, 1.05]; $P<0.001)$; (7) $\log _{10}$ D-dimer per mg/l increase $(\mathrm{HR}=3.00[2.17,4.16] ; P<0.001)$; and (8) $\log _{10}$ serum creatinine $p e r \mu \mathrm{mol} / \mathrm{L}$ increase $(\mathrm{HR}=4.55$ [2.72, 7.62]; $P<0.001$ ). In piecewise linear regression analyses $\log _{10} \mathrm{NLR}$ the interval from $\geq 0.4$ to $\leq 1.0$ was significantly associated with an increased risk of death. Our data identify covariates associated with risk of in hospital death in persons with COVID-19.
\end{abstract}

\section{Introduction}

The SARS-CoV-2 (severe acute respiratory syndrome coronavirus-2) pandemic has caused many deaths from coronavirus disease 2019 (COVID-19) [1-8]. The outbreak began in December 2019 in Wuhan city, Hubei province, China [9-16]. There are several studies or risk

These authors contributed equally: Lei Chen, Jianming Yu, Wenjuan $\mathrm{He}$, Li Chen, Guolin Yuan, Fang Dong, Wenlan Chen, Yulin Cao, Jingyan Yang, Liling Cai

Qiubai Li

qiubaili@hust.edu.cn

$\triangle \mathrm{Yu} \mathrm{Hu}$

dr_huyu@126.com

1 Institute of Hematology, Union Hospital, Tongji Medical College, Huazhong University of Science and Technology, Wuhan 430022, China

2 Department of Hematology, Wuhan Central Hospital, Tongji Medical College, Huazhong University of Science and Technology, Wuhan, China

3 Department of Hematology, Xiangyang Central Hospital, the Affiliated Hospital of Hubei University of Arts and Science, Xiangyang 441021, China factors for death from COVID-19 but most have relatively few subjects and come from 1 or 2 centers [17-26]. We analyzed prognostic covariates for death in 1859 subjects with confirmed COVID-19 from 7 centers in Wuhan city from January 20 to April 4, 2020. Curiously, we found COVID-19 progressed similarly until day 10 after admission but progressed more slowly in the cohort of subjects who died compared with those recovering, possibly because of therapy interventions $[27,28]$. Subjects who died had a greater frequency of comorbidities before COVID-19 and complications after developing COVID-19. We were able to show a

4 Intensive Care Units of Wuhan Third Hospital, Tongren Hospital of Wuhan University, Wuhan, China

5 Department of Urology Surgery, Union Hospital, Tongji Medical College, Huazhong University of Science and Technology, Wuhan 430022, China

6 Department of Hematology, General Hospital of Central Theater Command, PLA, Wuhan 300700, China

7 Wuhan Jin-Yin-Tan Hospital, Wuhan, China

8 Medical Records Statistics Department of Wuhan Third Hospital, Tongren Hospital of Wuhan University, Wuhan, China

9 Center for Hematology Research, Department of Immunology and Inflammation, Imperial College London, London, UK 
correlation between likelihood of death and several hematological and other laboratory covariates at diagnosis.

\section{Methods}

\section{Subjects}

From 20 January to 4 April 2020, all consecutive patients $\geq 18$ years were enrolled from Union Hospital (main part, Union West Hospital and Union Tumor Hospital), Wuhan Central Hospital, General Hospital of Central Theater Command, PLA, Wuhan Third Hospital and Wuhan JinYin-Tan Hospital. These hospitals were reconstructed and designated as COVID-19 treatment centers. Between February 4 and February 18, 2020 persons with clinical symptoms and a lung computed tomography (CT) scan consistent with COVID-19 were diagnosed as having COVID-19 without confirmation of SARS-CoV-2infection by quantitative reverse transcript polymerase chain reaction (qRT-PCR). After hospitalization subjects were tested by qRT-PCR to confirm the diagnosis and monitor their course. Beginning 4 March, 2020, antiSARS-CoV-2 IgM and/or IgG antibodies were assayed at Union Hospital and Wuhan Central Hospital by the centers to confirm the diagnosis and to evaluate suspected cases of COVID-19 which were qRT-PCR-negative [29]. Subjects in whom we could not confirm SARS-CoV-2infection by a qRT-PCR, IgM/IgG assay, or either were excluded from the study. Subjects recovering from COVID-19 were discharged and transferred to designated hotels, Fangcang shelter hospitals [30] or Leishenshan Hospital for 2-4 weeks of isolation or further care if needed. The study was approved by the Ethics Committees of Union Hospital (2020-0095) and of Wuhan Central Hospital (2020-007). Written and orally informed consent from subjects was waived by the Ethics Committees.

\section{Data collection}

We obtained epidemiological, demographic, clinical, laboratory, radiological, therapy, and outcomes data from electronic medical records using a standardized data collection form. Therapies included antibiotics, anti-viral therapy, corticosteroids, and supportive care including supplemental oxygen, mechanical ventilation (with and without intubation), and extracorporeal membrane oxygenation (ECMO). Data were independently entered and cross validated by two physicians (WH and JY). A third researcher $(\mathrm{QL})$ adjudicated discordances. Missing data were retrieved from the relevant hospital.

\section{SARS-CoV-2 testing and laboratory covariants}

Methods for diagnosis of SARS-CoV-2-infection by qRTPCR are described [24]. Before January 11, 2020 testing was done by a few institutions such as the Chinese Center for Disease Control and Prevention. Beginning January 11, 2020, qRT-PCR testing was done at local Centres for Disease Control and Prevention and from January 27, 2020 in the study hospitals. Beginning March 4, $2020 \mathrm{IgM} / \mathrm{IgG}$ antibodies to SARS-CoV-2 were tested at Union Hospital and after March 5, 2020 in Wuhan Central Hospital. Nasopharyngeal swab specimens were obtained every other day if there was clinical improvement judged by clinical signs and symptoms and lung CT scan. Subjects recovering were discharged after $\geq 2$ negative qRT-PCR tests $>24 \mathrm{~h}$ apart. Studies on admission included a CBC and differential, biochemistry panel, coagulation profile, and tests of inflammation including C-reactive protein (CRP), procalcitonin, lactate dehydrogenase (LDH), and ferritin. All subjects had a lung CT scan.

\section{Definitions}

Exposure history was defined as exposure to persons with confirmed SARS-CoV-2-infection or visiting the Huanan Wholesale Seafood Market, possible origin site of the SARS-CoV-2 epidemic in Wuhan city. Smoking history was defined as current or former smoker (stopping $>5$ years ago) with exposure of $\geq 20$ cigarettes per day for $\geq 1$ year ( 1 pack year). Fever was defined as temperature $\geq 37.3^{\circ} \mathrm{C}$. Diagnosis of bacterial infection required $\geq 1$ positive culture or a positive antigen detection test. Acute kidney injury, acute respiratory distress syndrome (ARDS), and acute cardiac injury were diagnosed according to guidelines or as reported [24, 31, 32]. Liver damage was defined as more than 2x upper limit of normal. Severity of COVID-19 was classified as; (1) mild; (2) moderate; (3) severe; or (4) critical according to the Chinese management guideline for COVID-19 (version 7) [29, 33]. Recovery was defined as complete resolution of clinical signs and symptoms, normalization of the lung CT scan (if abnormal) and $\geq 2$ negative qRT-PCR tests for SARS-CoV-2. Subjects dying of unrelated causes were excluded from analyses of COVID-19-related deaths. Invasive and noninvasive mechanical ventilations were defined as mechanical ventilation with and without intubation.

\section{Statistical analysis}

Demographics and clinical covariates were presented using descriptive statistics with frequencies (percentage) for discrete variables and median (IQR) and range for continuous variables. Medians were compared using independent group 
Fig. 1 Study flow diagram.

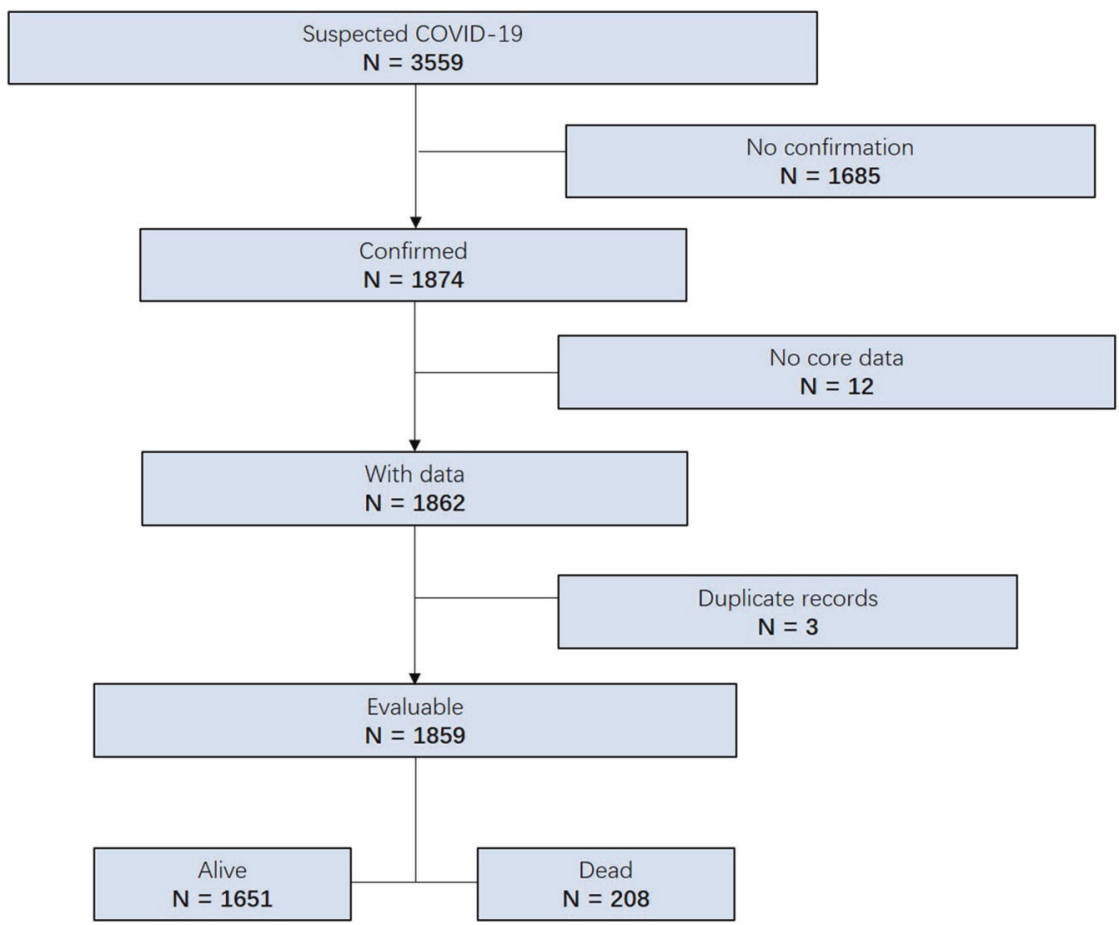

$t$ test and Mann-Whitney test for normally and abnormally distributed data. Proportions for categorical variables were compared by $\chi^{2}$ test. Missing data were ignored without multiple imputations. Covariates considered for correlations with death included age, sex, occupation, signs and symptoms, laboratory and radiological findings, smoking history, comorbidities including arterio-sclerotic cardio-vascular disease (ASCVD), arterial hypertension, diabetes mellitus, chronic obstructive pulmonary disease (COPD), cancer, ARDS, infection, septic shock, acute renal failure, myocardial infarction, liver injury, gastro-intestinal bleeding, disseminated vascular coagulation, and multiple organ failure. Neutrophil-to-lymphocyte ratio (NLR), D-dimer and serum creatine (Scr) were $\log _{10}$ transformed before the analyses because of non-normal distributions. Uni- and multi-variable Cox regression models were used to evaluate associations of covariants with risk of death. $\mathrm{R}$ version 3.5.2 was used for statistical analyses. For unadjusted comparisons, a two-sided alpha of $<0.05$ was considered significant. Analyses were not adjusted for multiple comparisons.

\section{Results}

\section{Admission clinical covariates}

From January 20 to April 4, 2020, 3559 subjects who died or had been discharged with clinical- or qRT-PCR- confirmed COVID-19 [34, 35] were enrolled. As shown in Fig. 1, 1859 subjects were included for analysis, with SARS-CoV-2-infection confirmed in 1790 subjects by qRT-PCR and by antibody testing in 69. Of the 1859 subjects, 208 died (11\%), 157 (8\%) recovered and discharged but remained hospitalized in other units for 2-4 weeks of recovery-related care, and 1494 (80\%) discharged and transferred to COVID-19 designated hotels or Fangcang shelter hospitals [30] for 2-4 weeks of isolation.

Median age was 59 years (Interquartile Range [IQR] 45-68 years; Table 1). 806 (43\%) were 60-79 years and $122(7 \%)>80$ years. 934 subjects were male $(50 \%) .111$ $(6 \%)$ were current or former smokers, $71(5 \%)$, health care provider, and $14(1 \%)$, with pregnancy or puerperium. In total, 4 subjects were exposed at Huanan Seafood Wholesale Market and $78(4 \%)$ had close contact with persons with confirmed SARS-CoV-2 infection. 579 (31\%) had hypertension, 268 (14\%), ASCVD, 262 (14\%), diabetes, 98 (5\%), gastro-intestinal disease, $69(4 \%)$, cancer and 61 (3\%), COPD. Most common signs and symptoms included fever $(n=1448,78 \%)$, shortness of breath $(n=716,39 \%)$, dry $(n=619,43 \%)$ or wet cough $(n=715,39 \%)$, fatigue $(n=695,37 \%)$, chills $(n=281,19 \%)$ and myalgia $(n=$ $315,17 \%)$. Bilateral pneumonia $(n=1570,88 \%)$ and Ground-glass opacity $(n=1331,75 \%)$ were two most findings in lung CT scan. 34 (2\%) subjects had mild, 1170 (63\%), moderate, 453 (24\%), severe and 202 (11\%) critical COVID-19. 
Table 1 Demographic and clinical covariates.

\begin{tabular}{|c|c|c|c|c|}
\hline & Total $n=1859$ & Alive $n=1651$ & Died $n=208$ & $P$ value \\
\hline Age, median (IQR), years & $59(45,68)$ & $57(43,66)$ & $70(63,78)$ & $<0.001$ \\
\hline Age distribution & & & & $<0.001$ \\
\hline$<40$ years & $342(18)$ & $337(20)$ & $5(2)$ & \\
\hline $40-59$ years & $589(32)$ & $556(34)$ & $33(16)$ & \\
\hline $60-79$ years & $806(43)$ & $681(41)$ & $125(60)$ & \\
\hline$\geq 80$ years & $122(7)$ & $77(5)$ & $45(22)$ & \\
\hline Female sex & $925(50)$ & $870(53)$ & $55(26)$ & $<0.001$ \\
\hline Smoking history & $111(6)$ & $86(5)$ & $25(13)$ & $<0.001$ \\
\hline Former smoker & $66(4)$ & $54(3)$ & $12(6)$ & \\
\hline Current smoker & $45(2)$ & $32(2)$ & $13(7)$ & \\
\hline Health care provider & $71(5)$ & $69(6)$ & $2(1)$ & 0.008 \\
\hline Pregnancy/Puerperium & $14(1)$ & $14(1)$ & $0(0)$ & 0.394 \\
\hline Exposure history & & & & 0.005 \\
\hline Huanan Seafood Market & $4(0.2)$ & $1(0.1)$ & $3(1)$ & \\
\hline Close contact with patients & $78(4)$ & $72(4)$ & $6(3)$ & \\
\hline \multicolumn{5}{|l|}{ Comorbidity } \\
\hline ASCVD & $268(14)$ & $205(12)$ & $63(30)$ & $<0.001$ \\
\hline Hypertension & $579(31)$ & 475 (29) & $104(50)$ & $<0.001$ \\
\hline Diabetes & $262(14)$ & $203(12)$ & $59(28)$ & $<0.001$ \\
\hline COPD & $61(3)$ & $49(3)$ & $12(6)$ & 0.039 \\
\hline Cancer & $69(4)$ & $52(3)$ & $17(8)$ & $<0.001$ \\
\hline Chronic kidney disease & $45(2)$ & $25(2)$ & $20(10)$ & $<0.001$ \\
\hline Gastro-intestinal disease & $98(5)$ & $82(5)$ & $16(8)$ & 0.097 \\
\hline Auto-immune disease & $10(1)$ & $9(1)$ & $1(1)$ & 0.999 \\
\hline Psychiatric disorders & $7(0.5)$ & $6(0.5)$ & $1(1)$ & 0.586 \\
\hline \multicolumn{5}{|l|}{ Signs and symptoms } \\
\hline Fever $^{\mathrm{a}}$ & $1448(78)$ & $1274(77)$ & $174(84)$ & 0.025 \\
\hline Temperature $\left({ }^{\circ} \mathrm{C}\right)^{\mathrm{b}}$ & $36.6(36.4,37.0)$ & $36.6(36.4,37.0)$ & $36.8(36.5,37.5)$ & $<0.001$ \\
\hline Shortness of breath & $716(39)$ & $572(35)$ & $144(70)$ & $<0.001$ \\
\hline Dry cough & $619(43)$ & $554(43)$ & $65(38)$ & 0.205 \\
\hline Wet cough & $715(39)$ & 609 (37) & $106(51)$ & $<0.001$ \\
\hline Fatigue & $695(37)$ & $595(36)$ & $100(48)$ & $<0.001$ \\
\hline Nausea or vomiting & $124(9)$ & $114(9)$ & $10(6)$ & 0.186 \\
\hline Diarrhea & $243(13)$ & $213(13)$ & $30(14)$ & 0.522 \\
\hline Chills & $281(19)$ & $236(18)$ & $45(26)$ & 0.015 \\
\hline Rhinorrhea & $33(2)$ & $30(2)$ & $3(1)$ & 0.999 \\
\hline Myalgia & $315(17)$ & $282(17)$ & $33(16)$ & 0.681 \\
\hline Headache & $107(6)$ & $101(6)$ & $6(3)$ & 0.061 \\
\hline \multicolumn{5}{|l|}{ Radiological features } \\
\hline Bilateral pneumonia & $1570(88)$ & $1402(87)$ & $168(96)$ & $<0.001$ \\
\hline Consolidation & $326(18)$ & $266(17)$ & $60(35)$ & $<0.001$ \\
\hline Ground-glass opacity & $1331(75)$ & $1213(76)$ & $118(69)$ & 0.042 \\
\hline Patchy shadows & $736(41)$ & $664(41)$ & $72(42)$ & 0.81 \\
\hline COVID-19 stage & & & & $<0.001$ \\
\hline Mild & $34(2)$ & $34(2)$ & $0(0)$ & \\
\hline Moderate & $1170(63)$ & $1162(70)$ & $8(4)$ & \\
\hline Severe & $453(24)$ & $427(26)$ & $26(13)$ & \\
\hline Critical & $202(11)$ & $28(2)$ & $174(84)$ & \\
\hline
\end{tabular}

Data are median (IQR) or $n(\%)$.

$A S C V D$ atherosclerotic cardio- and cerebro-vascular disease, COPD chronic obstructive pulmonary disease.

${ }^{\mathrm{a}} \geq 1$ temperature $\geq 37.3^{\circ} \mathrm{C}$ from onset of symptoms to admission.

${ }^{\mathrm{b}}$ Admission temperature. 


\section{Comparison of survivors and nonsurvivors by clinical covariates}

Subjects who died were older (medians 70 versus 57 years; $P<0.001$, Table 1$)$, more likely male $(74 \%$ versus $47 \%$; $P<0.001)$, more likely smokers $(13 \%$ versus $5 \%$; $P<$ $0.001)$ and less likely health care providers (1\% versus $6 \%$, $P=0.008)$. More subjects who died were exposure at the Huanan Seafood Wholesale Market ( $1 \%$ versus $0.1 \%, P=$ $0.005)$. Nonsurvivors more likely to have comorbidities of hypertension (50\% versus $29 \% ; P<0.001)$, ASCVD $(30 \%$ versus $12 \% ; P<0.001)$, diabetes $(28 \%$ versus $12 \% ; P<$ $0.001)$, COPD (6\% versus $3 \% ; P=0.039)$, cancer $(8 \%$ versus $3 \% ; P<0.001)$ and kidney failure $(10 \%$ versus $2 \%$; $P<0.001)$. Fever from illness onset to admission $(84 \%$ versus $77 \% ; P=0.025)$, shortness of breath $(70 \%$ versus $35 \% ; P<0.001)$, wet cough $(51 \%$ versus $37 \% ; P<0.001)$, fatigue ( $48 \%$ versus $36 \%$; $P<0.001$ ), chills ( $26 \%$ versus $18 \%$; $P=0.015$ ), bilateral pneumonia (96\% versus $87 \%$; $P<0.001)$ and lung consolidation on CT scan (35\% versus $17 \% ; P<0.001)$ were more common in subjects who died. Paradoxically, survivors were more likely to have groundglass lung opacity on lung CT scan (76\% versus $69 \% ; P=$ 0.042). Subjects who died were more likely to have critical COVID-19 on admission (84\% versus 2\%; $P<0.001$ ) and less likely to have moderate severity ( $4 \%$ versus $70 \%$; $P<0.001)$.

\section{Comparison of survivors and nonsurvivors by laboratory covariates}

There were significant differences in admission laboratory covariates between survivors and nonsurvivors (Table 2). Subjects who died had higher median neutrophils $(7 \times 10 \mathrm{E}$ $+9 / \mathrm{L}[\mathrm{IQR} 4-10 \times 10 \mathrm{E}+9 / \mathrm{L}]$ versus $3 \times 10 \mathrm{E}+9 / \mathrm{L}[\mathrm{IQR}$ $2-4 \times 10 \mathrm{E}+9 / \mathrm{L}] ; P<0.001)$, lower median lymphocytes $(0.6 \times 10 \mathrm{E}+9 / \mathrm{L}$ [IQR $0.4-0.9 \times 10 \mathrm{E}+9 / \mathrm{L}]$ versus median $1.2 \times 10 \mathrm{E}+9 / \mathrm{L}$ [IQR $0.9-1.6 \times 10 \mathrm{E}+9 / \mathrm{L}] ; P<$ $0.001)$, lower median platelets $(163 \times 10 \mathrm{E}+9 / \mathrm{L}$ [IQR $113-223 \times 10 \quad \mathrm{E}+9 / \mathrm{L}]$ versus $207 \quad 10 \mathrm{E}+9 / \mathrm{L} \quad[\mathrm{IQR}$ $161-268 \times 10 \mathrm{E}+9 / \mathrm{L}] ; \quad P<0.001)$ and higher median neutrophil-to-lymphocyte ratios (NLR; 11 [IQR 6-20] versus 3 [IQR 2-4]; $P<0.001)$. Nonsurvivors had lower median proportions of CD3-positive cells (60\% [IQR 52-70\%] versus 72\% [IQR 63-79\%]; $P<0.001$ ), median proportions of CD8-positive cells (16\% [IQR 11-20\%] versus $24 \%$ [IQR $18-30 \%$ ]; $P<0.001$ ), median proportions of NK-cells (8\% [IQR 3-12\%] versus 10\%, [IQR 6-17\%]; $P=0.011)$, and higher proportions of B-lymphocyte $(15 \%$ [IQR 9-28\%] versus 12\% [IQR 9-17\%]; $P=0.033$ ) and higher median CD4/CD8 ratios (3 [IQR 2-4] versus 2 [IQR $1-3] ; P<0.001)$ compared with survivors.
Subjects who died had longer median aPTT (37 s [IQR $31-42 \mathrm{~s}$ ] versus $34 \mathrm{~s}$ [IQR $30-38 \mathrm{~s}$; $P<0.001)$ and higher median concentrations of fibrinogen (4.3 g/L [IQR 3.2-5.1 $\mathrm{g} / \mathrm{L}]$ versus $3.7 \mathrm{~g} / \mathrm{L} \quad[\mathrm{IQR} \quad 2.9-4.6 \mathrm{~g} / \mathrm{L}] ; P<0.001)$ and median D-dimer concentrations $(2.5 \mathrm{mg} / \mathrm{L}$ [IQR $0.7-8 \mathrm{mg} / \mathrm{L}$ ] versus $0.4 \mathrm{mg} / \mathrm{L}$ [IQR $0.2-0.8 \mathrm{mg} / \mathrm{L}] ; P<0.001$ ).

Subjects who died also had higher median hCRP concentrations $(10 \mathrm{mg} / \mathrm{L} \quad[\mathrm{IQR} \quad 10-80 \mathrm{mg} / \mathrm{L}]$ versus $3 \mathrm{mg} / \mathrm{L}$ [IQR $1-10 \mathrm{mg} / \mathrm{L}] ; P<0.001)$, median procalcitonin concentrations $(0.3 \mathrm{ng} / \mathrm{ml}$ [IQR $0.1-0.6 \mathrm{ng} / \mathrm{mL}$ ] versus $0.05 \mathrm{ng} /$ $\mathrm{ml}$ [IQR $0.04-0.1 \mathrm{ng} / \mathrm{mL}$ ]; $P<0.001$ ), median LDH activities (412 U/L [IQR 306-561 U/L] versus $201 \mathrm{U} / \mathrm{L}$ [IQR 165-261 U/L]; $P<0.001$ ), median ferritin concentrations $(1579 \mathrm{ng} / \mathrm{mL}$ [IQR $1206-2000 \mathrm{ng} / \mathrm{mL}$ ] versus $470 \mathrm{ng} / \mathrm{mL}$ [IQR $197-940 \mathrm{ng} / \mathrm{mL}$ ]; $P<0.001)$, median IL-6 concentrations $(79 \mathrm{pg} / \mathrm{mL}$ [IQR $23-525 \mathrm{pg} / \mathrm{mL}$ ] versus $9 \mathrm{pg} / \mathrm{mL}$ [IQR $4-32 \mathrm{pg} / \mathrm{mL}$ ]; $P<0.001$ ), median IL-10 concentrations (10 $\mathrm{pg} / \mathrm{mL}$ [IQR $5-22 \mathrm{pg} / \mathrm{mL}$ ] versus $4 \mathrm{pg} / \mathrm{mL}[\mathrm{IQR} 3-5 \mathrm{pg} / \mathrm{mL}$ ]; $P<0.001)$ and lower median TNF- $\alpha$ concentrations $(2.7 \mathrm{pg} /$ $\mathrm{mL}$ [IQR $1.9-3.6 \mathrm{pg} / \mathrm{mL}$ ] versus $3.5 \mathrm{pg} / \mathrm{mL}$ [IQR $2.3-5.6$ $\mathrm{pg} / \mathrm{mL}] ; P=0.009$ ).

Subjects who died also had higher median activities of alanine aminotransferase (ALT; $58 \mathrm{U} / \mathrm{L}$ [IQR 30-139 U/L] versus $36 \mathrm{U} / \mathrm{L}$ [IQR $21-63 \mathrm{U} / \mathrm{L}] ; P<0.001)$, aspartate aminotransferase (AST; $64 \mathrm{U} / \mathrm{L}$ [IQR 40-140 U/L] versus $30 \mathrm{U} / \mathrm{L}$ [IQR 22-44 U/L]; $P<0.001)$, creatine kinase (262 U/L [IQR 135-636 U/L] versus $81 \mathrm{U} / \mathrm{L}$ [IQR $52-135 \mathrm{U} / \mathrm{L}] ; P<0.001)$ and median concentrations of total bilirubin $(24 \mu \mathrm{mol} / \mathrm{L}$ [IQR $15-36 \mu \mathrm{mol} / \mathrm{L}$ ] versus $13 \mu \mathrm{mol} / \mathrm{L}$ [IQR $10-18 \mu \mathrm{mol} / \mathrm{L}$ ]; $P<$ 0.001), b-type natriuretic peptide (BNP; $467 \mathrm{pg} / \mathrm{ml}$ [IQR $121-1467 \mathrm{pg} / \mathrm{ml}$ ] versus $47 \mathrm{pg} / \mathrm{ml}$, [IQR $15-140 \mathrm{pg} / \mathrm{ml}$ ]; $P<$ 0.001), myoglobin $(576 \mathrm{ng} / \mathrm{ml}$ [IQR $175-1013 \mathrm{ng} / \mathrm{ml}]$ versus $30 \mathrm{ng} / \mathrm{ml}$ [IQR $21-51 \mathrm{ng} / \mathrm{ml}] ; P<0.001)$ and troponin I (161 ng/L [IQR 46-712 ng/L] versus $3 \mathrm{ng} / \mathrm{L}$ [IQR $1-8 \mathrm{ng} / \mathrm{L}] ; P<$ 0.001 ), blood urea nitrogen (BUN; $15 \mathrm{mmol} / \mathrm{L}$ [IQR 9-27 $\mathrm{mmol} / \mathrm{L}]$ versus $5 \mathrm{mmol} / \mathrm{L}$ [IQR $4-6 \mathrm{mmol} / \mathrm{L}] ; P<0.001)$ and Scr $(108 \mu \mathrm{mol} / \mathrm{L} \quad[\mathrm{IQR} \quad 76-256 \mu \mathrm{mol} / \mathrm{L}]$ versus $69 \mu \mathrm{mol} / \mathrm{L}$ [IQR 58-81 $\mu \mathrm{mol} / \mathrm{L}] ; P<0.001)$.

\section{Complications and treatments for survivors and non-survivors}

Subjects who died were more likely to have complications (207 [99.5\%] versus 1039 [63\%]; $P<0.001$ ) including ARDS (174 [84\%] versus 53 [3\%]; $P<0.001$ ), bacterial infection (180 [87\%] versus 383 [23\%]; $P<0.001)$ and liver damage (102 [50\%] versus 354 [22\%]; $P<0.001$; Table 3). Nonsurvivors also had higher incidence of heart injury (130 [63\%] versus 99 [6\%]; $P<0.001)$, multiple organ failure (126 [61\%] versus $3[0.2 \%] ; P<0.001)$, acute kidney injury (82 [39\%] versus $17[1 \%] ; P<0.001)$, septic shock (63 [37\%] versus 1 $[0.1 \%] ; P<0.001)$, abnormal coagulation parameters (47 
Table 2 Laboratory covariates on admission.

\begin{tabular}{|c|c|c|c|c|c|}
\hline Covariates (normal range) & $N$ & Total & Alive & Died & $P$ value \\
\hline \multicolumn{6}{|l|}{ CBC } \\
\hline Neutrophils $\times 10 \mathrm{E}+9 / \mathrm{L}(1.8-6.3)$ & 1816 & $3(2,5)$ & $3(2,4)$ & $7(4,10)$ & $<0.001$ \\
\hline Lymphocytes $\times 10 \mathrm{E}+9 / \mathrm{L}(1.1-3.2)$ & 1847 & $1.0(0.8,1.6)$ & $1.2(0.9,1.6)$ & $0.6(0.4,0.9)$ & $<0.001$ \\
\hline Monocytes ×10 E + 9/L (0.1-0.6) & 1805 & $0.4(0.3,0.5)$ & $0.4(0.3,0.5)$ & $0.3(0.2,0.5)$ & $<0.001$ \\
\hline Hemoglobin, g/L (115-150) & 1433 & $128(117,139)$ & $128(117,139)$ & $129(117,140)$ & 0.539 \\
\hline Platelets $\times 10 \mathrm{E}+9 / \mathrm{L}(125-350)$ & 1814 & $203(155,264)$ & $207(161,268)$ & $163(113,223)$ & $<0.001$ \\
\hline NLR & 1814 & $3(2,5)$ & $3(2,4)$ & $11(6,20)$ & $<0.001$ \\
\hline \multicolumn{6}{|l|}{ Inflammation covariates } \\
\hline hCRP, mg/L $(<4)$ & 995 & $4(1,10)$ & $3(1,10)$ & $10(10,80)$ & $<0.001$ \\
\hline Procalcitonin, ng/ml $(<0.5)$ & 1643 & $0.06(0.05,0.1)$ & $0.05(0.04,0.1)$ & $0.3(0.1,0.6)$ & $<0.001$ \\
\hline LDH, U/L (109-245) & 1729 & $212(170,292)$ & $201(165,261)$ & $412(306,561)$ & $<0.001$ \\
\hline Ferritin, ng/ml (4.6-204) & 308 & $567(246,1218)$ & $470(197,940)$ & $1579(1206,2000)$ & $<0.001$ \\
\hline \multicolumn{6}{|l|}{ Coagulation covariates } \\
\hline aPTT, s (28-43.5) & 1356 & $34(30,38)$ & $34(30,38)$ & $37(31,42)$ & $<0.001$ \\
\hline Fibrinogen, g/L (2-4) & 1323 & $3.7(2.9,4.6)$ & $3.7(2.9,4.6)$ & $4.3(3.2,5.1)$ & $<0.001$ \\
\hline D-dimer, mg/L $(<0.5)$ & 1602 & $0.4(0.2,1.1)$ & $0.4(0.2,0.8)$ & $2.5(0.7,8)$ & $<0.001$ \\
\hline \multicolumn{6}{|l|}{ Biochemical covariates } \\
\hline ALT, U/L (5-35) & 1832 & $38(22,67)$ & $36(21,63)$ & $58(30,139)$ & $<0.001$ \\
\hline AST, U/L (8-40) & 1830 & $32(22,49)$ & $30(22,44)$ & $64(40,140)$ & $<0.001$ \\
\hline Total bilirubin, $\mu \mathrm{mol} / \mathrm{L}(5.1-19)$ & 1586 & $14(10,19)$ & $13(10,18)$ & $24(15,36)$ & $<0.001$ \\
\hline Creatine kinase, U/L (26-140) & 1493 & $88(54,165)$ & $81(52,135)$ & $262(135,636)$ & $<0.001$ \\
\hline BNP, pg/ml $(<100)$ & 838 & $61(18,242)$ & $47(15,140)$ & $467(121,1467)$ & $<0.001$ \\
\hline Myoglobin, ng/ml (<140) & 972 & $35(21,73)$ & $30(21,51)$ & $576(175,1013)$ & $<0.001$ \\
\hline Troponin I, ng/L (<26.2) & 1083 & $4(1,14)$ & $3(1,8)$ & $161(46,712)$ & $<0.001$ \\
\hline BUN, mmol/L (2.9-8.2) & 1815 & $5(4,7)$ & $5(4,6)$ & $15(9,27)$ & $<0.001$ \\
\hline $\mathrm{Scr}, \mu \mathrm{mol} / \mathrm{L}(44-106)$ & 1813 & $71(59,85)$ & $69(58,81)$ & $108(76,256)$ & $<0.001$ \\
\hline \multicolumn{6}{|l|}{ Lymphocyte subsets } \\
\hline $\mathrm{CD} 3+,(58-84 \%)$ & 759 & $71(62,78)$ & $72(63,79)$ & $60(52,70)$ & $<0.001$ \\
\hline $\mathrm{CD} 4+,(25-51 \%)$ & 759 & $41(32,48)$ & $41(33,48)$ & $37(28,46)$ & 0.094 \\
\hline $\mathrm{CD} 8+,(14-39 \%)$ & 759 & $23(17,30)$ & $24(18,30)$ & $16(11,20)$ & $<0.001$ \\
\hline NK cell, $(3-30 \%)$ & 561 & $10(6,16)$ & $10(6,17)$ & $8(3,12)$ & 0.011 \\
\hline B lymphocyte, (4-18\%) & 561 & $13(9,18)$ & $12(9,17)$ & $15(9,28)$ & 0.033 \\
\hline $\mathrm{CD} 4+/ \mathrm{CD} 8+$ Ratio $(0.41-2.72)$ & 755 & $2(1,3)$ & $2(1,3)$ & $3(2,4)$ & $<0.001$ \\
\hline \multicolumn{6}{|l|}{ Cytokines } \\
\hline IL-4, pg/ml (0.1-3.2) & 505 & $3(2,4)$ & $3(2,4)$ & $2(2,3)$ & 0.21 \\
\hline IL-6, pg/ml (0.1-2.9) & 857 & $10(4,42)$ & $9(4,32)$ & $79(23,525)$ & $<0.001$ \\
\hline IL-10, pg/ml (0.1-5) & 505 & $4(3,6)$ & $4(3,5)$ & $10(5,22)$ & $<0.001$ \\
\hline TNF- $\alpha, p g / m l(0.1-23)$ & 505 & $3.3(2.2,5.4)$ & $3.5(2.3,5.6)$ & $2.7(1.9,3.6)$ & 0.009 \\
\hline IFN- $\gamma, \mathrm{pg} / \mathrm{ml}(0.1-18)$ & 505 & $3.1(2.0,4.1)$ & $3.1(2.0,4.1)$ & $2.7(1.8,4.2)$ & 0.313 \\
\hline
\end{tabular}

Data are median (IQR).

$N L R$ neutrophil-to-lymphocyte ratio, $h C R P$ high-sensitivity c-reactive protein, $L D H$ lactate dehydrogenase, $a P T T$ activated partial thromboplastin time, $A L T$ alanine aminotransferase, AST aspartate aminotransferase, BNP B-type natriuretic peptide, BUN blood urea nitrogen, Scr serum creatinine, $I L$ interleukin, $T N F$ tumor necrosis factor, $I F N$ interferon.

[23\%] versus $2[0.1 \%] ; P<0.001)$ and gastro-intestinal bleeding (29 [14\%] versus 5 [0.3\%]; $P<0.001$ ).

Also, subjects who died were more likely to receive antibiotics (203 [98\%] versus 1356 [84\%]; $P<0.001)$, antifungal drugs (39 [20\%] versus 32 [2\%]; $P<0.001)$, lopinavir and ritonavir (59 [35\%] versus 280 [22\%]; $P<$ $0.001)$, corticosteroids (165 [80\%] versus 588 [36\%]; $P<$ 0.001), intravenous immunoglobin (IVIG; 105 [52\%] versus 401 [26\%]; $P<0.001)$, high-flow nasal cannula oxygen therapy (152 [89\%] versus 81 [6\%]; $P<0.001)$, 
Table 3 Complications and therapy.

Total $n=1859$ Alive $n=1651 \quad$ Died $n=208 \quad P$ value

(89)

(11)

\begin{tabular}{|c|c|c|c|c|}
\hline \multicolumn{5}{|l|}{ Complications } \\
\hline ARDS & $227(12)$ & $53(3)$ & $174(84)$ & $<0.001$ \\
\hline Bacterial infections & $563(30)$ & $383(23)$ & $180(87)$ & $<0.001$ \\
\hline Septic shock & $64(4)$ & $1(0.1)$ & $63(37)$ & $<0.001$ \\
\hline Acute kidney injury & $99(5)$ & $17(1)$ & $82(39)$ & $<0.001$ \\
\hline Cardiac injury & $229(12)$ & $99(6)$ & $130(63)$ & $<0.001$ \\
\hline Abnormal LFT & $456(25)$ & $354(22)$ & $102(50)$ & $<0.001$ \\
\hline Gastro-intestinal bleeding & $34(2)$ & $5(0.3)$ & $29(14)$ & $<0.001$ \\
\hline Coagulopathy & $49(3)$ & $2(0.1)$ & $47(23)$ & $<0.001$ \\
\hline Multiple organ failure & $129(7)$ & $3(0.2)$ & $126(61)$ & $<0.001$ \\
\hline \multicolumn{5}{|l|}{ Therapy } \\
\hline Antibiotics & $1559(85)$ & $1356(84)$ & $203(98)$ & $<0.001$ \\
\hline Antifungal drugs & $71(4)$ & $32(2)$ & $39(20)$ & $<0.001$ \\
\hline Oseltamivir & $757(41)$ & $688(42)$ & $69(33)$ & 0.021 \\
\hline Umifenovir & $1386(75)$ & $1226(74)$ & $160(77)$ & 0.351 \\
\hline Lopinavir/Ritonavir & $339(23)$ & $280(22)$ & $59(35)$ & $<0.001$ \\
\hline Interferon & $387(21)$ & $344(21)$ & $43(21)$ & 0.957 \\
\hline Corticosteroids & $753(41)$ & $588(36)$ & $165(80)$ & $<0.001$ \\
\hline IVIG & $506(29)$ & $401(26)$ & $105(52)$ & $<0.001$ \\
\hline High-flow nasal cannula oxygen therapy & $233(16)$ & $81(6)$ & $152(89)$ & $<0.001$ \\
\hline Noninvasive mechanical ventilation & $145(8)$ & $27(2)$ & $118(57)$ & $<0.001$ \\
\hline Invasive mechanical ventilation & $85(5)$ & $12(1)$ & $73(35)$ & $<0.001$ \\
\hline ECMO & $4(0.2)$ & $1(0.1)$ & $3(1)$ & 0.005 \\
\hline CRRT & $23(2)$ & $4(0.3)$ & $19(11)$ & $<0.001$ \\
\hline \multicolumn{5}{|l|}{ Outcomes } \\
\hline ICU admission & $106(6)$ & $36(2)$ & $70(34)$ & $<0.001$ \\
\hline Time from illness onset to ICU admission, median (IQR), days & $14(10,20)$ & $14(10,21)$ & $14(10,20)$ & 0.962 \\
\hline ICU length of stay, median (IQR), days & $10(4,17)$ & $10(5,18)$ & $10(4,16)$ & 0.676 \\
\hline $\begin{array}{l}\text { Time from illness onset to repeated negative SARS-CoV-2 tests, median } \\
\text { (IQR), days }\end{array}$ & $22(17,28)$ & $22(17,28)$ & $21(15,27)$ & 0.284 \\
\hline Time from illness onset to admission, median (IQR), days & $10(7,15)$ & $10(7,16)$ & $9(6,12)$ & $<0.001$ \\
\hline Time from illness onset to progression, median (IQR), days & $10(7,15)$ & $10(6,14)$ & $12(9,18)$ & $<0.001$ \\
\hline Time from illness onset to outcome, median (IQR), days & $30(23,37)$ & $31(24,38)$ & $21(14,28)$ & $<0.001$ \\
\hline Time from diagnosis to outcome, median (IQR), days & $19(13,27)$ & $20(14,28)$ & $11(5,17)$ & $<0.001$ \\
\hline Time from admission to outcome, median (IQR), days & $18(12,23)$ & $18(14,23)$ & $10(6,19)$ & $<0.001$ \\
\hline
\end{tabular}

LFT liver function test, $A R D S$ acute respiratory distress syndrome, IVIG intravenous immunoglobin, ECMO extra-corporeal membrane oxygenation, $C R R T$ continuous renal replacement therapy, ICU intensive care unit.

noninvasive mechanical ventilation (118 [57\%] versus 27 [2\%]; $P<0.001)$, invasive mechanical ventilation (73 [35\%] versus $12[1 \%] ; P<0.001)$, ECMO (3 [1\%] versus $1[0.1 \%] ; P=0.005)$ and continuous renal replacement therapy (CRRT) (19 [11\%] versus 4 [0.3\%]; $P<$ $0.001)$.

Nonsurvivors had briefer median intervals from onset of symptoms to admission (9 d [IQR 6-12 d] versus $10 \mathrm{~d}$ [IQR $7-16 \mathrm{~d}] ; P<0.001)$ and median intervals from onset of symptoms to death or discharge (21 d [IQR 14-28] versus
$31 \mathrm{~d}$ [IQR 24-38 d]; $P<0.001$ ), and from admission to death or discharge (10 d [IQR] 6-19 d versus $18 \mathrm{~d}$ [IQR 14-23 d]; $P<0.001)$ but longer median intervals from onset of symptoms to progression (median $12 \mathrm{~d}$ [IQR 9-18 d] versus $10 \mathrm{~d}[\mathrm{QR} 6-14 \mathrm{~d}] ; P<0.001)$. There were no differences between survivors and nonsurvivors in median intervals from symptoms onset to ICU admission (14 d [IQR $10-20 \mathrm{~d}$ ] versus $14 \mathrm{~d}$ [IQR $10-21 \mathrm{~d}] ; P=0.962)$ or median intervals to negative SARS-CoV-2 testing $(21 \mathrm{~d}$ [IQR $15-27 \mathrm{~d}$ ] versus $22 \mathrm{~d}$ [IQR $17-28 \mathrm{~d}] ; P=0.284)$. In 
Table 4 Risk factors for death.

$\begin{array}{lll}\begin{array}{l}\text { Uni-variable HR } \\ (95 \% \mathrm{CI})\end{array} & P \text { value } & \begin{array}{l}\text { Multivariable HR } \\ (95 \% \mathrm{CI})\end{array}\end{array}$

\begin{tabular}{|c|c|c|c|c|}
\hline \multicolumn{5}{|l|}{ Clinical covariates } \\
\hline Age, years & $1.07(1.06-1.08)$ & $<0.001$ & $1.04(1.03-1.06)$ & $<0.001$ \\
\hline Female sex (vs male) & $0.35(0.26-0.48)$ & $<0.001$ & .. & .. \\
\hline Smoking history (vs nonsmoking) & $2.43(1.59-3.73)$ & $<0.001$ & $1.84(1.17-2.92)$ & 0.009 \\
\hline Health care provider (vs non health care provider) & $0.24(0.06-0.96)$ & 0.044 & .. & .. \\
\hline \multicolumn{5}{|l|}{ Comorbidity (Yes/No) } \\
\hline ASCVD & $2.56(1.90-3.45)$ & $<0.001$ & .. & .. \\
\hline Diabetes & $2.47(1.82-3.34)$ & $<0.001$ & .. & .. \\
\hline Hypertension & $2.21(1.68-2.90)$ & $<0.001$ & .. & .. \\
\hline Cancer & $2.59(1.58-4.26)$ & $<0.001$ & .. & .. \\
\hline \multicolumn{5}{|l|}{ Symptoms and complications (Y/N) } \\
\hline Dyspnea & $6.26(4.76-8.24)$ & $<0.001$ & .. & .. \\
\hline Wet cough & $1.63(1.24-2.14)$ & 0.001 & .. & .. \\
\hline ARDS & $54.21(37.13-79.14)$ & $<0.001$ & .. & .. \\
\hline Bacterial infections & $18.36(12.16-27.72)$ & $<0.001$ & .. & .. \\
\hline Temperature at admission $\left({ }^{\circ} \mathrm{C}\right)$ & $1.50(1.28-1.75)$ & $<0.001$ & $1.32(1.07-1.64)$ & 0.009 \\
\hline \multicolumn{5}{|l|}{ Laboratory covariates } \\
\hline Neutrophils $\times 10 \mathrm{E}+9 / \mathrm{L}$ & $1.23(1.20-1.26)$ & $<0.001$ & .. & .. \\
\hline Lymphocytes $x 10 \mathrm{E}+9 / \mathrm{L}$ & $0.07(0.05-0.10)$ & $<0.001$ & .. & .. \\
\hline $\log _{10}$ NLR & $1.06(1.06-1.07)$ & $<0.001$ & $3.30(2.10-5.19)$ & $<0.001$ \\
\hline Platelets $\times 10 \mathrm{E}+9 / \mathrm{L}$ & $0.99(0.99-1.00)$ & $<0.001$ & $0.996(0.994-0.998)$ & 0.001 \\
\hline hCRP, mg/L & $1.02(1.02-1.02)$ & $<0.001$ & .. & .. \\
\hline Procalcitonin, ng/ml & $1.23(1.18-1.28)$ & $<0.001$ & .. &.. \\
\hline $\mathrm{LDH}, \mathrm{U} / \mathrm{L}$ & $1.00(1.00-1.00)$ & $<0.001$ & .. &.. \\
\hline Ferritin, ng/ml & $1.00(1.00-1.00)$ & $<0.001$ & .. & .. \\
\hline aPTT, s & $1.06(1.04-1.08)$ & $<0.001$ & $1.04(1.02-1.05)$ & $<0.001$ \\
\hline $\log _{10}$ D-dimer, mg/L & $1.09(1.08-1.11)$ & $<0.001$ & $3.00(2.17-4.16)$ & $<0.001$ \\
\hline Total bilirubin, $\mu \mathrm{mol} / \mathrm{L}$ & $1.03(1.03-1.04)$ & $<0.001$ & .. &.. \\
\hline Creatine kinase, U/L & $1.00(1.00-1.00)$ & $<0.001$ & .. &.. \\
\hline Troponin I, ng/L & $1.00(1.00-1.00)$ & $<0.001$ & .. &.. \\
\hline $\mathrm{BUN}, \mathrm{mmol} / \mathrm{L}$ & $1.06(1.05-1.07)$ & $<0.001$ &.. &.. \\
\hline $\log _{10} \operatorname{Scr}, \mu \mathrm{mol} / \mathrm{L}$ & $1.00(1.00-1.00)$ & $<0.001$ & $4.55(2.72-7.62)$ & $<0.001$ \\
\hline IL-6 & $1.00(1.00-1.00)$ & $<0.001$ & .. & .. \\
\hline IL-10 & $1.00(1.00-1.00)$ & 0.004 & .. &.. \\
\hline CD3-positive, $\%$ & $0.95(0.93-0.97)$ & $<0.001$ & .. &.. \\
\hline CD8-positive, $\%$ & $0.91(0.88-0.94)$ & $<0.001$ & .. &.. \\
\hline $\mathrm{CD} 4 / \mathrm{CD} 8$ ratio & $1.27(1.18-1.37)$ & $<0.001$ & .. &.. \\
\hline
\end{tabular}

$C I$ confidence interval, $A S C V D$ atherosclerotic cardio- and cerebro-vascular disease, $A R D S$ acute respiratory distress syndrome, $N L R$ neutrophil-tolymphocyte ratio, $h C R P$ high-sensitive c-reactive protein, $L D H$ lactate dehydrogenase, $a P T T$ activated partial thromboplastin time, $B U N$ blood urea nitrogen, $S c r$ serum creatinine.

total, 178 of the 208 subjects who died $(86 \%)$ had a positive qRT-PCR test until death.

\section{Risk factors for death}

In total, 33 covariants had significant associations with risk of death in uni-variable analyses, 8 of which remained significant in multi-variable analyses including age $(\mathrm{HR}=$ 1.04 [1.03, 1.06]; $P<0.001)$, smoking history (HR $=1.84$ $[1.17,2.92] ; P=0.009)$, temperature value $\left({ }^{\circ} \mathrm{C}\right)$ at admission $(\mathrm{HR}=1.32$ [1.07-1.64]; $P=0.009), \log _{10}$ NLR (HR $=3.30[2.10,5.19] ; P<0.001)$, admission platelet concentration $(\mathrm{HR}=0.996$; $[0.994-0.998] ; P=0.001)$, aPTT on admission $(\mathrm{HR}=1.04[1.02,1.05] ; P<0.001), \log _{10}$ 

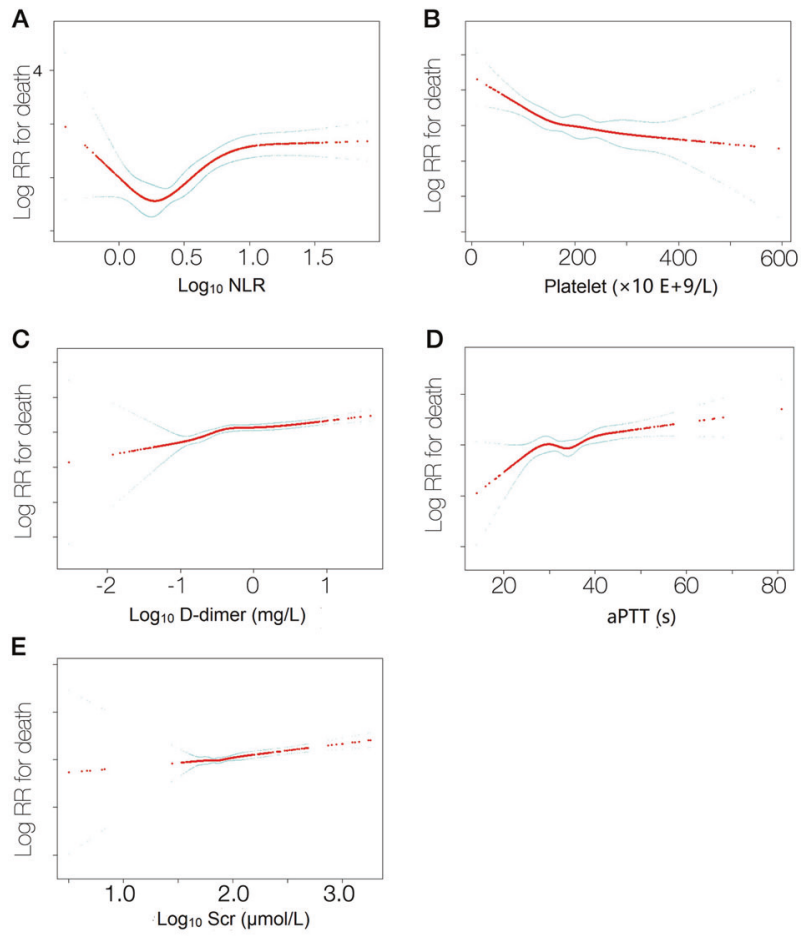

Fig. 2 The linear relationship between admission covariants and risk of death. (a) $\log 10 \mathrm{NLR}$, (b) platelet (x $10 \mathrm{E}+9 / \mathrm{L}$ ), (c) $\log 10 \mathrm{D}-$ dimer $(\mathrm{mg} / \mathrm{L})$, (d) aPTT (s) and (e) $\log 10 \mathrm{Scr}(\mu \mathrm{mol} / \mathrm{L})$.

D-dimer $(\mathrm{HR}=3.00[2.17,4.16] ; P<0.001)$, and $\log _{10} \mathrm{Cr}$ $(\mathrm{HR}=4.55$ [2.72, 7.62]; $P<0.001$; Table 4).

We further showed the linear relationship between these covariants except age, smoking history, and temperature at admission and risk of death (Fig. 2). Based on the steep curve of $\log _{10}$ NLR we conducted a further piecewise linear regression analysis of NLR and death. The results indicate a $\log _{10}$ NLR value of $\geq 0.4$ to $\leq 1.0$ is significantly associated with risk of death (Table 5).

\section{Discussion}

We identified eight hospital admission covariates which are independent risk factors for death in almost 2000 persons with COVID-19 including older age, smoking history, higher body temperature $\left({ }^{\circ} \mathrm{C}\right)$, and levels of D-dimer, aPTT, Scr, platelet, and NLR on admission. Several are reported by others; however, we were unable to confirm other risk factors reported in smaller datasets [15, 18-21, 23, 24, 26, 36].

We identified $\log _{10}$ NLR as an independent risk factor for death with an $\mathrm{HR}=14.1(3.2,61.2)$ with $\log _{10}$ values $\geq 0.4$ to $\leq 1.0$ but not otherwise (Table 5). Although higher neutrophil and lower lymphocyte concentrations and higher NLR were previously reported [23, 26, 36-38], $\log _{10} \mathrm{NLR}$ has not.
Table 5 Piecewise linear regression analysis of the effect of Neutrophil-to-lymphocyte ratio (NLR) on risk of death.

\begin{tabular}{lcr}
\hline & Hazard ratio (95\% CI) & $P$ value \\
\hline $\log _{10}$ NLR & $3.30(2.10-5.19)$ & $<0.001$ \\
$\log _{10}$ NLR $<0.4$ & $0.44(0.02-10.09)$ & 0.608 \\
$\log _{10}$ NLR $\geq 0.4, \leq 1.0$ & $14.06(3.23-61.21)$ & $<0.001$ \\
$\log _{10}$ NLR $>1.0$ & $0.49(0.17-1.44)$ & 0.195 \\
\hline
\end{tabular}

There are important limitations to our study. First, not all covariates were available in all subjects including body mass index and SOFA score (a factor for death identified by logistic regression analysis) [24]. Also, BNP and TNI were reported in different units and were therefore not included for multi-variable Cox regression analyses. Second, at the data lock $65(1.8 \%)$ subjects remained hospitalized and are excluded from our analyses. Third, some covariates such as bacterial coinfection and BUN could not be accurately analysed and interpreted together with other covariants for interactions. Our conclusions although based on a large dataset require confirmation. Nevertheless, they may be useful in predicting outcomes in persons with COVID-19.

\section{Data availability}

All data generated or analyzed are included in this typescript including supplement.

Acknowledgements We thank participating patients, families, and health care providers. Funded by the National Natural Science Foundation of China (NSFC; 81974009 to QL and 81974221 to ZC) and the Fundamental Research Funds for the Central Universities (2020kfyXGYJ086 to QL). RPG acknowledges support from the National Institute of Health Research (NIHR) Biomedical Research Centre funding scheme.

Author contributions $\mathrm{YH}$ and QL designed the study. LeC, JY, WH, LCh, GY, FD, WC, YC, JY, LC, DW, QR, LL, QL, WR, FG, HW, and $\mathrm{ZC}$ collected the data. All authors had full access to the data, were involved in data interpretation and vouch for the accuracy of the analyses. QL, LeC, and RPG prepared the typescript which all authors approved final approval and supported the decision to submit for publication.

\section{Compliance with ethical standards}

Conflict of interest The authors declare that they have no conflict of interest.

Publisher's note Springer Nature remains neutral with regard to jurisdictional claims in published maps and institutional affiliations.

\section{References}

1. WHO. Coronavirus disease (COVID-2019) situation report. 2020 2020/02/27: https://www.who.int/docs/default-source/coronaviruse/ 
situation-reports/20200517-covid-20200519-sitrep-20200118.pdf? sfvrsn $=20200521 \mathrm{c} 20200510$ dafe_20200518. Accessed 18 May 2020.

2. Richardson S, Hirsch JS, Narasimhan M, Crawford JM, McGinn $\mathrm{T}$, Davidson KW, et al. Presenting characteristics, comorbidities, and outcomes among 5700 patients hospitalized with COVID-19 in the New York City Area. JAMA. 2020: https://doi.org/10.1001/ jama.2020.6775.

3. Grasselli G, Pesenti A, Cecconi M. Critical care utilization for the COVID-19 outbreak in Lombardy, Italy: early experience and forecast during an emergency response. JAMA. 2020;323:1545-6.

4. Grasselli G, Zangrillo A, Zanella A, Antonelli M, Cabrini L, Castelli A, et al. Baseline characteristics and outcomes of 1591 patients infected with SARS-CoV-2 admitted to ICUs of the Lombardy Region, Italy. JAMA. 2020;323:1574-81.

5. Onder G, Rezza G, Brusaferro S. Case-fatality rate and characteristics of patients dying in relation to COVID-19 in Italy. JAMA. 2020;323:1775-6.

6. MacLaren G, Fisher D, Brodie D. Preparing for the most critically Ill patients with COVID-19: the potential role of extracorporeal membrane oxygenation. JAMA. 2020;323:1245-6.

7. Bhatraju PK, Ghassemieh BJ, Nichols M, Kim R, Jerome KR, Nalla AK, et al. Covid-19 in critically Ill patients in the Seattle Region - Case Series. N Engl J Med. 2020: https://doi.org/10. 1056/NEJMoa2004500.

8. Wang C, Horby PW, Hayden FG, Gao GF. A novel coronavirus outbreak of global health concern. Lancet. 2020;395:470-3.

9. Zhu N, Zhang D, Wang W, Li X, Yang B, Song J, et al. A novel Coronavirus from patients with Pneumonia in China, 2019. N Engl J Med. 2020;382:727-33.

10. Li Q, Guan X, Wu P, Wang X, Zhou L, Tong Y, et al. Early transmission dynamics in Wuhan, China, of novel Coronavirus-infected Pneumonia. N Engl J Med. 2020;382: 1199-207.

11. Guan W-J, Ni Z-Y, Hu Y, Liang W-H, Ou C-Q, He J-X, et al. Clinical characteristics of Coronavirus disease 2019 in China. N Engl J Med. 2020;382:1708-20.

12. Huang $\mathrm{C}$, Wang $\mathrm{Y}$, Li X, Ren $\mathrm{L}$, Zhao J, Hu Y, et al. Clinical features of patients infected with 2019 novel coronavirus in Wuhan, China. Lancet. 2020;395:497-506.

13. Chen N, Zhou M, Dong X, Qu J, Gong F, Han Y, et al. Epidemiological and clinical characteristics of 99 cases of 2019 novel coronavirus pneumonia in Wuhan, China: a descriptive study. Lancet. 2020;395:507-13.

14. Wang D, Hu B, Hu C, Zhu F, Liu X, Zhang J, et al. Clinical characteristics of 138 hospitalized patients with 2019 novel Coronavirus-infected Pneumonia in Wuhan, China. JAMA. 2020;323:1061-9.

15. Wu Z, McGoogan JM. Characteristics of and important lessons from the Coronavirus disease 2019 (COVID-19) outbreak in China: summary of a report of 72314 cases from the Chinese Center for Disease Control and Prevention. JAMA. 2020;323: 1239-42.

16. Li W, Wang D, Guo J, Yuan G, Yang Z, Gale RP, et al. COVID19 in persons with chronic myeloid leukaemia. Leukemia. 2020: https://doi.org/10.1038/s41375-020-0853-6.

17. Du Y, Tu L, Zhu P, Mu M, Wang R, Yang P, et al. Clinical features of 85 fatal cases of COVID-19 from Wuhan: a retrospective observational study. Am J Respir Crit Care Med. 2020: https://doi.org/10.1164/rccm.202003-200543OC.

18. Chen T, Wu D, Chen H, Yan W, Yang D, Chen G, et al. Clinical characteristics of 113 deceased patients with coronavirus disease 2019: retrospective study. BMJ. 2020;368:m1091.

19. Jordan RE, Adab P, Cheng KK. Covid-19: risk factors for severe disease and death. BMJ. 2020;368:m1198.
20. Du R-H, Liang L-R, Yang C-Q, Wang W, Cao T-Z, Li M, et al. Predictors of mortality for patients with COVID-19 Pneumonia caused by SARS-CoV-2: a prospective cohort study. Eur Respir J. 2020: https://doi.org/10.1183/13993003.13900524-13992020.

21. Liang W-H, Guan W-J, Li C-C, Li Y-M, Liang H-R, Zhao Y, et al. Clinical characteristics and outcomes of hospitalised patients with COVID-19 treated in Hubei (epicenter) and outside Hubei (non-epicenter): a Nationwide Analysis of China. Eur Respir J. 2020: https://doi.org/10.1183/13993003.13900562-13992020.

22. Tu W-J, Cao J, Yu L, Hu X, Liu Q. Clinicolaboratory study of 25 fatal cases of COVID-19 in Wuhan. Intensive Care Med. 2020: https://doi.org/10.1007/s00134-00020-06023-00134.

23. Wu C, Chen X, Cai Y, Xia JA, Zhou X, Xu S, et al. risk factors associated with acute respiratory distress syndrome and death in patients with Coronavirus Disease 2019 Pneumonia in Wuhan, China. JAMA Intern Med. 2020: https://doi.org/10.1001/jama internmed.2020.0994.

24. Zhou F, Yu T, Du R, Fan G, Liu Y, Liu Z, et al. Clinical course and risk factors for mortality of adult inpatients with COVID-19 in Wuhan, China: a retrospective cohort study. Lancet. 2020;395: 1054-62.

25. Fang L, Karakiulakis G, Roth M. Are patients with hypertension and diabetes mellitus at increased risk for COVID-19 infection? Lancet Resp Med. 2020;8:e21.

26. Zhang G, Zhang J, Wang B, Zhu X, Wang Q, Qiu S. Analysis of clinical characteristics and laboratory findings of 95 cases of 2019 novel coronavirus pneumonia in Wuhan, China: a retrospective analysis. Respir Res. 2020;21:1-10.

27. Li H, Chen $\mathrm{C}, \mathrm{Hu} \mathrm{F}$, Wang J, Zhao Q, Gale RP, et al. Impact of corticosteroid therapy on outcomes of persons with SARS-CoV-2, SARS-CoV, or MERS-CoV infection: a systematic review and meta-analysis. Leukemia. 2020;34:1503-11.

28. Gale RP Perspective: SARS-CoV-2, COVID-19 and haematologists. Acta Haematol. 2020: https://doi.org/10.1159/000508021.

29. National Health Commission of China. Chinese Clinical Guidance for COVID-19 pneumonia Diagnosis and Treatment (7th version). 2020: http://www.nhc.gov.cn/yzygj/s7653p/202003/202046c209 294a202007dfe202004cef202080dc202007f205912eb201989. shtml. Accessed 10 March 2020.

30. Chen S, Zhang Z, Yang J, Wang J, Zhai X, Bärnighausen T, et al. Fangcang shelter hospitals: a novel concept for responding to public health emergencies. Lancet. 2020;395:1305-14.

31. Lameire NH, Bagga A, Cruz D, De Maeseneer J, Endre Z, Kellum JA, et al. Acute kidney injury: an increasing global concern. Lancet. 2013;382:170-9.

32. Force* TADT. Acute respiratory distress syndrome: the Berlin definition. JAMA. 2012;307:2526-33.

33. He W, Chen L, Chen L, Yuan G, Fang Y, Chen W, et al. COVID19 in persons with haematological cancers. Leukemia. 2020: https://doi.org/10.1038/s41375-41020-40836-41377.

34. National Health Commission of China. The novel coronavirus pneumonia diagnosis and treatment program, 5th version. 2020: http://www.nhc.gov.cn/yzygj/s7653p/202002/202003b202009 b202894ac202009b204204a202079db202005b208912d204440. shtml. Accessed 05 April 2020.

35. WHO. Clinical management of severe acute respiratory infection when novel coronavirus $(\mathrm{nCoV})$ infection is suspected: interim guidance. 2020: https://www.who.int/publications-deta il/clinical-management-of-severe-acute-respiratory-infection-w hen-novel-coronavirus-(ncov)-infection-is-suspected. Accessed 5 April.

36. Zhao X, Zhang B, Li P, Ma C, Gu J, Hou P, et al. Incidence, clinical characteristics and prognostic factor of patients with COVID-19: a systematic review and meta-analysis. medRxiv 2020: https://doi.org/10.1101/2020.1103.1117.20037572. 
37. Liang W, Liang H, Ou L, Chen B, Chen A, Li C, et al. Development and validation of a clinical risk score to predict the occurrence of critical illness in hospitalized patients with COVID-19. JAMA Intern Med. 2020: https://doi.org/10.1007/s00134-00020-06023-00134.
38. Liu J, Li S, Liu J, Liang B, Wang X, Wang H, et al. Longitudinal characteristics of lymphocyte responses and cytokine profiles in the peripheral blood of SARS-CoV-2 infected patients. EBioMedicine. 2020;55:102763. 